Roberto Geraldo Baruzzi 1

Vera Lucia de Barros 2

Douglas Rodrigues 1

Ana Lucia Medeiros de Souza 1

Heloisa Pagliaro ${ }^{1}$

\section{Saúde e doença em índios Panará (Kreen-Akarôre) após vinte e cinco anos de contato com o nosso mundo, com ênfase na ocorrência de tuberculose (Brasil Central)}

\author{
Health and disease among Panará \\ (Kreen-Akarôre) Indians in Central Brazil after \\ twenty-five years of contact with our World, \\ with an emphasis on tuberculosis
}

1 Departamento de Medicina Preventiva, Escola Paulista de Medicina, Universidade Federal de São Paulo. Rua Botucatu 740, São Paulo, SP 04023-900, Brasil. baruzzi@medprev.epm.br 2 Centro Universitário São Camilo. Av. Nazaré 1501, São Paulo, $S P$ 04263-200, Brasil.

\begin{abstract}
The Panará, who had previously lived in isolation from Brazilian national society in the Amazon forest, were first contacted in 1973. Two years later they were moved to another area in Central Brazil. During this same period they were reduced to 82 members, the survivors of a population of 400 to 500 in the mid-1960s. In 1995 they returned to a small area in their old territory still not occupied by outsiders. There, three years later, a health survey showed a presumed diagnosis of tuberculosis in 15 individuals out of a population of 181. Further tests in the town of Colider, based on clinical data and chest X-rays, confirmed the diagnosis in 10 Panará (6 children under 10 years of age and 4 adults from 40 to 50 years old). BCG scars were present in the entire population. The nutritional status of Panará children was better than that of other indigenous groups in the Amazon region. The following measures were introduced for Tb control: a) treatment follow-up in the village, under direct supervision by both a nurse and the local indigenous health worker; $b$ ) compliance with defined criteria for ending treatment; $c$ ) periodic control of contacts and non-contacts; $c$ ) and establishment of a reference system with the health services in Colider.
\end{abstract}

Key words Nutrition Disorders; Tuberculosis; Panará; South American Indians

Resumo Em 1973, houve a quebra do estado de isolamento dos Panará (Kren-Akarore) no interior da floresta amazônica. Dois anos após estavam reduzidos a 82 indivíduos, de uma população estimada em 400 a 500 em meados dos anos 60. Em 1998, o exame dos Panará, nas cabeceiras do rio Iriri, sul do Pará, levou ao diagnóstico presuntivo de tuberculose em 15 indivíduos, dos quais 10 foram confirmados na cidade de Colider com base em dados clínicos e radiológicos. Desses 10 casos, 6 eram menores de 10 anos de idade e 4 tinham de 40 a 50 anos. Todos da tribo apresentavam cicatriz vacinal do BCG. Em crianças, a prevalência de desnutrição crônica e de anemia ferropriva foi menor do que a relatada em outros grupos indígenas da região amazônica. As medidas de controle da Tb, a nível local, incluíram: a) continuidade do tratamento dos pacientes, na aldeia, sob supervisão do Auxiliar de Enfermagem e do Agente Indígena de Saúde; b) observância dos critérios de cura; c) acompanhamento clínico de comunicantes e não-comunicantes dada a elevada prevalência da doença; d) implantação de sistema de referência e contra-referência com serviços de saúde de Colider.

Palavras-chave Transtornos Nutricionais; Tuberculose; Panará; Índios Sul-Americanos 


\section{Introdução}

Nos últimos anos, a incidência e a mortalidade por tuberculose no país têm aumentado, atingindo 90.000 casos novos e 6.000 óbitos em 1997 (MS, 1999). A situação tende a ser mais grave nas comunidades indígenas face às maiores dificuldades de acesso aos serviços de saúde e de controle. Especial atenção deve ser dada a grupos indígenas de contato mais recente com a nossa sociedade, sujeitos ao risco de rápida propagação da infecção pelo bacilo de Koch a partir dos primeiros contatos com fontes de infecção.

Em 1973, os índios Panará (ou Kreen-Akarôre, como eram então referidos) foram contatados na região do rio Peixoto de Azevedo, Brasil Central, onde viviam isolados no interior da floresta. Chegava ao fim um processo de aproximação que se estendera por alguns anos e que provocou elevada mortalidade. De uma população estimada em 400 a 500 indivíduos, em meados da década de 1960, os Panará estavam reduzidos a 140 a 150 por ocasião do contato e, dois anos após, a 82, época em que se deu a abertura da estrada Cuiabá-Santarém, que cortava o território tribal (Heelas, 1979). Face ao risco de total aniquilamento, os remanescentes foram levados para o Parque Indígena do Xingu (PIX). Ao ingressarem no Parque, em janeiro de 1975, foram examinados por uma equipe médica da Escola Paulista de Medicina e do Serviço Nacional de Tuberculose, sendo feita a pesquisa de BK no escarro e aplicado o BCG intradérmico em adultos e crianças (Baruzzi et al., 1977).

Foi difícil a adaptação ao novo habitat, e o convívio com as 16 tribos culturalmente diversas que habitavam o PIX. Novos óbitos ocorreram nos primeiros meses para, a seguir, iniciarse um processo de lento crescimento populacional. Após permanecerem no PIX por cerca de 20 anos, os Panará decidiram voltar ao antigo território tribal, sonho que sempre acalentaram. No extremo norte desse território, nas cabeceiras do rio Iriri, encontraram uma área ainda não ocupada por cidades, fazendas ou garimpos e construíram ali sua nova aldeia: Nacipotire (Arnt et al., 1998). O acesso à aldeia foi assegurado pela abertura de um pequeno campo de pouso, mas as dificuldades de transporte tornaram menos freqüente a presença de profissionais da saúde, inclusive por parte da Escola Paulista de Medicina/Universidade Federal de São Paulo (EPM/UNIFESP), que desenvolve um programa de saúde no PIX desde 1965 (Baruzzi et al., 1978).

Em julho de 1998, na aldeia Nacipotire, procedeu-se ao levantamento das condições de saúde dos Panará, do estado nutricional das crianças e à busca ativa de casos de tuberculose, com a adoção de medidas de controle desta doença.

\section{Material e métodos}

\section{População estudada}

Os Panará pertencem ao tronco lingüístico Macro-Jê. A aldeia Nacipotire, de coordenadas geográficas 9o30' S e 5415’ W, está situada nas cabeceiras do rio Iriri, no sul do Pará, em uma área de floresta de 495.000 hectares, demarcada pelo governo federal em 1996. A população, em julho de 1998, era de 181 indivíduos da mesma etnia; apenas um indivíduo deixou de participar do inquérito por estar ausente na ocasião.

\section{Metodologia empregada}

O inquérito teve por base a ficha médica que foi introduzida em 1975 e usada no trabalho de campo, na qual constam dados de identificação, registros clínicos, exames anteriores e vacinas aplicadas. No exame físico foram seguidos os procedimentos propedêuticos habituais. As medidas antropométricas - peso e altura - foram obtidas de forma padronizada. $\mathrm{Na}$ aferição do peso foi utilizada a balança portátil microeletrônica da marca Kraus-Cas com precisão de $100 \mathrm{~g}$. Na aferição da estatura em crianças com menos de dois anos de idade foi feita a medida do comprimento com o emprego da régua antropométrica pediátrica; nos demais grupos etários foi utilizada a fita métrica Raven Minimetre com precisão de $0,1 \mathrm{~cm}$. Na avaliação do estado nutricional das crianças menores de 10 anos foram empregados os índices antropométricos altura para a idade e peso para altura, expressos em escore $\mathrm{z}$ do padrão NCHS/WHO (WHO, 1986). Foram classificadas como desnutridas crônicas as crianças apresentando valores do indicador altura para idade abaixo de -2 escore $\mathrm{z}$ e, com sobrepeso, aquelas com valores do indicador peso para altura acima de +2 escore $z$. O cálculo dos índices antropométricos foi realizado com o auxilio do programa EPINUT (Columbier et al., 1991).

Os diagnósticos de anemia e do grau de severidade foram baseados nos valores de referência para a concentração de hemoglobina recomendados pela Organização Mundial da Saúde segundo a idade (WHO, 1989). O nível crítico de concentração de hemoglobina indi- 
cativo de anemia foi de $11 \mathrm{~g} / \mathrm{dl}$ para as crianças de 6 meses a 5 anos de idade e de $12 \mathrm{~g} / \mathrm{dl}$ dos 6 aos 14 anos. A anemia foi considerada leve quando a concentração de hemoglobina foi superior a $10 \mathrm{~g} / \mathrm{dl}$ e inferior ao valor crítico; moderada, quando menor ou igual a $10 \mathrm{~g} / \mathrm{dl}$ e maior ou igual a $7 \mathrm{~g} / \mathrm{dl}$; e severa, quando a concentração foi inferior a $7 \mathrm{~g} / \mathrm{dl}$. Na dosagem da hemoglobina foi utilizado o Fotômetro Hemocue, de procedência sueca.

Em sintomáticos respiratórios foi feita a pesquisa do bacilo de Koch no escarro, em lâminas coradas pelo método de Ziehl. Ao encerrar o trabalho de campo, a relação dos casos suspeitos de tuberculose foi entregue aos profissionais de saúde locais a serem encaminhados à cidade de Colider, no norte de Mato Grosso - centro de referência médica para os Panará-, para exames complementares.

\section{Aspectos éticos}

O presente estudo insere-se no programa de saúde que a EPM/UNIFESP desenvolve no PIX e visa fornecer subsídios para as ações de saúde e sua avaliação. Antes de dar início ao exame dos índios Panará, o objetivo do trabalho e os procedimentos a serem adotados foram explicados, ficando bem explícito o caráter voluntário da participação e a liberdade de o indivíduo deixar de participar a qualquer momento que desejasse. A equipe médica, por sua vez, comprometeu-se a encaminhar os resultados do estudo para a comunidade. Estes esclarecimentos foram prestados inicialmente aos líderes comunitários e ao Agente Indígena de Saúde local e, depois, para toda a comunidade. Não houve nenhuma recusa em participar do projeto, tendo sido firmado um termo de adesão pelas lideranças indígenas com o concurso do Agente Indígena de Saúde.

\section{Resultados}

A Tabela 1 mostra a distribuição etária dos índios Panará nos anos 1975, 1995 e 1998. Com taxa de crescimento médio de $4,9 \%$ ao ano, a população Panará, em 1998, atingiu 181 indivíduos.

A Tabela 2 apresenta a prevalência de desnutrição em crianças menores de 10 anos, com desnutrição crônica presente nos três subgrupos etários considerados, identificada a partir de retardo no crescimento, enquanto que o déficit pondero-estatural foi observado apenas em $1,3 \%$ das crianças (ou seja, em duas crianças do grupo etário de 24 a 59 meses). A ocor-
Tabela 1

Estrutura etária (\%) dos índios Panará, nos anos de 1975, 1995 e 1998.

\begin{tabular}{lrrr}
\hline Grupos Etários & \multicolumn{3}{c}{ Anos } \\
& $\begin{array}{c}1975 \\
\mathrm{n}=79\end{array}$ & $\begin{array}{c}1995 \\
\mathrm{n}=162\end{array}$ & $\begin{array}{c}1998 \\
\mathrm{n}=181\end{array}$ \\
\hline $0-9$ & 31,7 & 47,5 & 42,0 \\
$10-19$ & 31,6 & 24,2 & 29,4 \\
$20-29$ & 24,0 & 9,9 & 9,4 \\
$30-39$ & 8,9 & 6,8 & 5,5 \\
$40-49$ & 3,8 & 6,8 & 8,8 \\
$50-59$ & 0,0 & 3,0 & 4,4 \\
60 e mais & 0,0 & 1,8 & 0,5 \\
Total & 100,0 & 100,0 & 100,0 \\
\hline
\end{tabular}

Tabela 2

Prevalência (\%) de desnutrição e de sobrepeso em crianças menores de 10 anos. Índios Panará, 1998

\begin{tabular}{llccc}
\hline Idade (meses) & $\mathbf{n}$ & \multicolumn{2}{c}{$\begin{array}{c}\text { Desnutrição1 } \\
\text { Peso } \\
\text { para idade }\end{array}$} & $\begin{array}{l}\text { Sobrepeso } \\
\text { para altura } \\
\text { Peso } \\
\text { para altura }\end{array}$ \\
\hline $0-23$ & 18 & 16,7 & 0 & 11,1 \\
$24-59$ & 20 & 10,0 & 5,0 & 10,0 \\
$60-119$ & 37 & 10,8 & 0 & 5,4 \\
Total & 75 & 12,0 & 1,3 & 8,0 \\
\hline
\end{tabular}

1 Desnutrição - índice altura para idade e peso para altura inferior a -2 z score.

2 Sobrepeso - índice peso para altura superior a +2 z score.

rência de sobrepeso foi verificada em $8 \%$ das crianças.

Anemia, conforme os critérios adotados, foi observada em $56,1 \%$ das crianças menores de 14 anos de idade, nas formas leves e moderadas (Tabela 3).

Ao exame físico foi constatada a presença da cicatriz vacinal do BCG em todos os adultos e crianças. Gânglios linfáticos cervicais e axilares aumentados, mas raramente ultrapassando 0,5 a $1 \mathrm{~cm}$ de diâmetro, sem sinais inflamatórios, foram palpáveis em $11 \%$ das crianças menores de cinco anos, em $23 \%$ entre 5 e 10 anos e em $7,5 \%$ entre 10 e 15 anos. Não foi constatada a presença de esplenomegalia na população. Com base em dados clínicos-propedêuticos foi feito o diagnóstico presuntivo de tuberculose em 15 casos, sendo negativa a pesquisa de BK no escarro, no campo. Na ocasião, foi solicitado o encaminhamento desses casos à ci- 
Tabela 3

Prevalência (\%) de anemia ferropriva e grau de severidade em crianças de seis meses a quatorze anos de idade. Índios Panará, 1998.

\begin{tabular}{lccccc}
\hline \multirow{2}{*}{ Idade } & $\mathbf{n}$ & \multicolumn{4}{c}{ Anemia (\%) } \\
& & Leve & Moderada & Severa & Total \\
\hline 6 meses a 5 anos & 36 & 22,2 & 11,1 & 0 & 33,3 \\
6 a 14 anos & 46 & 67,4 & 6,5 & 0 & 73,9 \\
Total & 82 & 47,6 & 8,5 & 0 & 56,1 \\
\hline
\end{tabular}

Valores normais de hemoglobina (WHO): de 6 meses a 5 anos $\geq 11 \mathrm{~g} / \mathrm{dl}$, de 6 a 14 anos $\geq 12 \mathrm{~g} / \mathrm{dl}$. diagnóstico de Esplenomegalia Hiperreativa da Malária, anteriormente denominada Síndrome de Esplenomegalia Tropical (Marsden \& Crane, 1976).

Em 1981 foram diagnosticados e tratados os primeiros casos de tuberculose com comprometimento pulmonar entre os Panará. No decorrer do tempo, outros casos foram sendo diagnosticados, para tal situação concorrendo as dificuldades encontradas na implantação de medidas de controle seja na supervisão do tratamento e fornecimento regular da medicação, seja na observância dos critérios de cura e na vigilância epidemiológica dos comunicantes. A baixa positividade do exame de escarro para o bacilo de Koch em áreas indígenas (Nutels, 1968) tende a dificultar a confirmação diagnóstica em casos suspeitos, que é agravada pelas dificuldades de acesso à radiografia do tórax.

O levantamento de 1998 a respeito das condições de saúde dos Panará incluiu inquérito nutricional em crianças menores de dez anos de idade, das quais $12 \%$ apresentaram déficit na altura para a idade e $1,3 \%$ na relação peso para a altura. Fagundes Neto et al. (1981), em inquérito realizado de 1974 a 1976 na população infantil do Alto Xingu - região sul do Parque Indígena do Xingu -, encontraram déficit pondero-estatural de grau 1 em menos de $5 \%$ das crianças e déficit de grau 2 só em 1,3\% das crianças no ano de 1974 . Ainda, na região do Alto Xingu, em inquérito de 1992, Mattos (1996) encontrou déficit de altura para idade em $20 \%$ das crianças. Em índios Suruí, do estado de Rondônia, cujo contato se reporta a 1969, Coimbra Jr. \& Santos (1991) encontraram, em crianças menores de nove anos de idade, $46,3 \%$ com baixa estatura para a idade e $6,6 \%$ de desnutrição pela relação peso/altura. Os inquéritos citados corroboram os resultados observados por outros autores em grupos indígenas do Brasil no que se refere à presença freqüente de déficit de altura para a idade (Santos, 1993).

O mesmo perfil antropométrico foi observado no ano de 1996 em inquérito de amplitude nacional voltado para a população infantil de área rural: $10,5 \%$ de déficit de estatura e 2,3\% de déficit ponderal (Monteiro, 1997).

Em 1975, ao ingressarem no PIX, 68\% das crianças Panará menores de dez anos de idade apresentaram valores de hemoglobina inferiores a $11 \mathrm{~g} / \mathrm{dl}$, em contraposição a 42\% em 1998. Neste último inquérito, 33\% das crianças de 6 meses a 5 anos de idade apresentaram anemia (Tabela 3). Segundo Coimbra Jr. \& Santos (1991), a prevalência de anemia foi de $70 \%$ em crianças Suruí menores de 5 anos de idade. No mesmo grupo etário, a presença de anemia em, res- 
pectivamente, $46,9 \%, 43,9 \%$ e $46,4 \%$ das crianças foi registrada em inquéritos realizados nas cidades de São Paulo, Recife e Salvador (Monteiro et al., 1999). Estes percentuais são superiores aos observados nas crianças Panará de mesmo grupo etário.

Deve-se destacar que, no presente inquérito, foi adotado o valor de $12 \mathrm{~g} / \mathrm{dl}$ como ponto de corte para a hemoglobina para o grupo etário de 6 a 14 anos, segundo recomendação da OMS (Organização Mundial de Saúde), o que deve ser tomado em conta ao analisar a alta prevalência de anemia encontrada neste grupo etário $(74 \%)$.

A avaliação das condições de saúde dos Panará não incluiu a pesquisa de parasitas intestinais. Cabe mencionar que, em 1975, fora evidenciado parasitismo por Ascaris lumbricoides em $15 \%$ dos examinados e, por ancilostomídeos, em todos, exceto um (Baruzzi et al., 1977). Em anos mais recentes, Anaruma Filho (1995) encontrou parasitismo intestinal por A. lumbricoides em $60 \%$ e por ancilostomídeos em $20 \%$ dentre os menores de dez anos de idade. Embora não seja possível estabelecer a eventual correlação entre o grau de anemia e a presença de parasitismo por esses helmintos para cada criança, deve ser considerada a ação espoliadora dos mesmos nas condições de nutrição e anemia na população infantil.

A partir de dados da anamnese e do exame físico foi feito o diagnóstico presuntivo de tuberculose em 15 indivíduos, tendo sido negativos os exames de escarro para a pesquisa do BK. Foi relativamente freqüente o encontro de gânglios linfáticos discretamente aumentados: cervicais e axilares, bem como, por vezes, inguinais, na população infantil, o que sugere, na ausência de outras etiologias, reação à infecção pelo bacilo de Koch.

Em Colider foi confirmado o diagnóstico de tuberculose em 2/3 dos casos suspeitos, na maioria com base no exame clínico e na radiografia do tórax, uma vez que o exame de escarro foi positivo somente em dois casos, em adultos. Deixam de ser comentados os achados radiológicos por não termos tido acesso às radio- grafias e tampouco aos respectivos relatórios. Esta é uma situação que ocorre com freqüência nas áreas indígenas: a falta de informações adequadas a respeito dos pacientes que são enviados para centros urbanos com a finalidade de elucidação diagnóstica e tratamento.

Ao analisar a elevada prevalência da tuberculose nos Panará duas ordens de fatores devem ser consideradas. Primeiro, o tipo de habitação pouco ensolarada e sem divisões internas, a proximidade das redes de dormir distribuídas por grupos familiares, situação agravada, algumas vezes, pelo número relativamente elevado de moradores. Em segundo lugar, o deslocamento dos Panará - iniciado em 1995 e completado no ano seguinte - para local distante do PIX, somente alcançado por via aérea, o que prejudicou as ações de saúde e o controle da tuberculose.

Fica demonstrado nos Panará que, a despeito da ampla cobertura vacinal com o BCG intradérmico, a tuberculose pode assumir características epidêmicas na falta de medidas de controle adequadas. No entanto, como pudemos observar nas tribos do PIX, praticamente deixam de ocorrer formas graves da tuberculose: miliar e meníngea na população infantil, como ocorria antes da introdução do BCG intradérmico.

A grave situação da tuberculose entre os $\mathrm{Pa}$ nará exige a concentração de esforços por parte dos órgãos assistenciais, com a supervisão do tratamento dos pacientes, observância dos critérios de cura e controle dos comunicantes. No presente inquérito ficou realçada, no trabalho de campo, a importância do exame físico sistemático para o levantamento das condições de saúde-doença da população.

A avaliação nutricional das crianças Panará mostrou a presença de formas crônicas de desnutrição e anemia em proporções menores ao observado em alguns inquéritos realizados em outros grupos indígenas, não existindo elementos suficientes para se afirmar que exista associação, naquela tribo, entre a elevada prevalência da tuberculose e o estado nutricional.

\section{Agradecimentos}

Ao Enor Mantovani, auxiliar de enfermagem, Eduardo N. Canó, médico-residente e Sucren, Agente Indígena de Saúde, da aldeia Nacipotire, pela colaboração no trabalho de campo; ao Waldir Luiz Alves e Fabio T. Montesano, do Departamento de Medicina Preventiva, pelo auxílio prestado, e aos Panará pela amável acolhida e participação. 


\section{Referências}

ANARUMA FILHO, F., 1995. Parasitoses Intestinais, por Helmintos, em Índios "Agricultores" e "Caçadores-coletores". Parque Indígena do Xingu, Brasil Central. Dissertação de Mestrado, São Paulo: Universidade Estadual de Campinas.

ARNT, R.; PINTO, L. F.; PINTO, R. \& MARTINELLI, P., 1998. Panará, a Volta dos Índios Gigantes. São Paulo: Instituto Socioambiental.

BARUZZI, R. G.; MARCOPITO, L. F. \& IUNES, M., 1978. Programa médico preventivo da Escola Paulista de Medicina no Parque Nacional do Xingu. Revista de Antropologia, 21:155-171.

BARUZZI, R. G.; MARCOPITO, L. F.; SERRA, M. L. C.; SOUZA, F. A. A. \& STABILE, C., 1977. The KreenAkarôre: A recently contacted indigenous tribe. Ciba Foundation Symposium, 49 (new series):179211.

COIMBRA Jr., C. E. A. \& SANTOS, R. V., 1991. Avaliação do estado nutricional num contexto de mudança sócio-econômica: o grupo indígena Suruí do Estado de Rondônia, Brasil. Cadernos de Saúde Pública, 7:538-562.

COLUMBIER, D; DIONISIUS, J P; DESVÉ, G., ET AL, 1991. Epinut: A software for analysis of nutrition survey. Version 1. Paris: EPICENTRE.

FAGUNDES NETO, U.; BARUZZI, R. G.; WEHBA, J.; SILVESTRINI, W. S.; MORAIS, M. B. \& CAINELLI, M., 1981. Observations of the Alto Xingu indians (Central Brazil) with special reference to nutritional evaluation in children. American Journal of Clinical Nutrition, 34:2.229-2.235.

HEELAS, R. H., 1979. The Organization of the Panará, $a$ Gê Tribe of Central Brazil. Ph. D. Thesis, Oxford: University of Oxford.

MARSDEN, P. D. \& CRANE, G. G., 1976. The Tropical Esplenomegaly Syndrome. A Current Appraisal. Revista do Instituto de Medicina Tropical de São Paulo, 18:54-70.
MATTOS, A. P., 1996. Avaliação do Estado Nutricional das Crianças Índias do Alto Xingu Utilizando Critérios Antropométricos Dependentes da Idade. Dissertação de Mestrado, São Paulo: Escola Paulista de Medicina, Universidade Federal de São Paulo.

MONTEIRO, C. A., 1997. O Panorama da Nutrição Infantil nos Anos 90. Brasília: Fundo de Nações Unidas para a Infância.

MONTEIRO, C. A.; SZAFARC, S. C. \& MONDINI, L., 1999. Tendência secular da anemia. In: Como $e$ por que Melhoram (ou Pioram) os Indicadores de Saúde e Nutrição na Infância? O Caso da Cidade de São Paulo na Segunda Metade do Século XX. Relatório Técnico. São Paulo: Núcleo de Pesquisas Epidemiológicas em Nutrição e Saúde, Universidade de São Paulo.

MS (Ministério da Saúde), 1999. Plano Nacional de Controle da Tuberculose. Brasília: Fundação Nacional de Saúde, Ministério da Saúde.

NUTELS, N., 1968. Medical Problems of Newly Contacted Indian Group. In: Biomedical Challenges Presented by the American Indian (Pan American Health Organization, ed.), Scientific Publication 165, pp. 68-76, Washington, DC: PAHO.

SANTOS, R. V., 1993. Crescimento Físico e Estado Nutricional de Populações Indígenas Brasileiras. $\mathrm{Ca}$ dernos de Saúde Pública, 9 (Sup.1):46-57.

WHO (World Health Organization), 1986. Use and Interpretation of Anthropometric Indicators of Nutritional Status. Bulletin of the World Health Organization, 64:929-941. Geneva: WHO.

WHO (World Health Organization), 1989. Preventing and Controlling Iron Deficiency Anaemia through Primary Health Care. A Guide for Health Administrators and Program Managers. Geneva: WHO. 